\title{
Construction and activity analyses of single functional mouse peroxiredoxin 6 (Prdx6)
}

\author{
Lu-Lu Wang ${ }^{1}$, Shi-Ying $\mathrm{Lu}^{1}$, Pan $\mathrm{Hu}^{1}$, \\ Bao-Quan $\mathrm{Fu}^{2}$, Yan-Song $\mathrm{Li}^{1}$, Fei-Fei Zhai ${ }^{1}$, Dan-Di Ju${ }^{1}$, \\ Shi-Jun Zhang ${ }^{1}$, Bing Su${ }^{1}$, Yu Zhou ${ }^{1}$, Zeng-Shan Liu ${ }^{1}$, Hong-Lin Ren ${ }^{1}$ \\ ${ }^{1}$ Key Laboratory of Zoonosis Research, Institute of Zoonosis/College of Veterinary Medicine, \\ Jilin University, Xi An Da Lu 5333, Changchun 130062, China \\ ${ }^{2}$ State Key Laboratory of Veterinary Etiological Biology, \\ Key Laboratory of Veterinary Public Health of the Ministry of Agriculture, \\ Key Laboratory of Veterinary Parasitology of Gansu Province, \\ Lanzhou Veterinary Research Institute, \\ Chinese Academy of Agricultural Sciences, Lanzhou 730046, China \\ renhl@jlu.edu.cn
}

Received: September 3, 2018 Accepted: January 14, 2019

\begin{abstract}
Introduction: Peroxiredoxin $6(\operatorname{Prdx} 6)$ is a bifunctional protein with glutathione peroxidase activity and phospholipase A2 activity. Previous studies have shown a significant positive correlation between the intracellular survival ability of Brucella and Prdx6. Here, the Prdx6 enzyme with a single activity was constructed to facilitate study of the relationship between the single function of Prdx6 and Brucella infection. Material and Methods: The target open reading frame (ORF) DNAs of Prdx6 with a single active centre were prepared using gene splicing by overlap extension PCR (SOE-PCR), and the recombinant eukaryotic expression plasmids inserted by Prdx6 with the single activity centre were constructed and transfected into murine Raw 264.7 macrophages. The glutathione peroxidase activity and phospholipase A2 activity of the constructed Prdx6 were examined. Results: The core centres $\left(\mathrm{Ser}^{32}\right.$ and $\mathrm{Cys}^{47}$ ) of Prdx6 were successfully mutated by changing the $94^{\text {th }}$ nucleotide from $\mathrm{T}$ to $\mathrm{G}$ and the $140^{\text {th }}$ nucleotide from $\mathrm{G}$ to $\mathrm{C}$ in the two enzyme activity cores, respectively. The constructed recombinant plasmids of Prdx6 with the single active centre were transfected into murine macrophages showing the expected single functional enzyme activity, which MJ33 or mercaptosuccinate inhibitors were able to inhibit. Conclusion: The constructed mutants of Prdx6 with the single activity cores will be a benefit to further study of the biological function of Prdx6 with different enzyme activity.
\end{abstract}

Keywords: mouse, peroxiredoxin 6, glutathione peroxidase, phospholipase A2, SOE-PCR.

\section{Introduction}

The peroxiredoxin (Prdx) family is a family of non-selenium-dependent peroxidases widely present in various tissues and organs of the body (4). The main physiological function of the Prdx family relating to various diseases such as tumours, those of the central nervous system, etc. is to eliminate free radicals in the body and reduce the damage they cause to the body (11). There are six subtypes of the Prdx family in mammals, which are classified into 1-Cys and 2-Cys depending on the number of cysteine residues. Peroxiredoxins 1, 2, 3, 4, and 5 belong to the 2-Cys subtype $(2,4)$. Peroxiredoxin 6 (Prdx6) belonging to the 1-Cys subtype is $40 \%$ conserved with other Prdxs (13). Differing from other Prdx family members, Prdx6 has a single cysteine residue, which determines why the catalytic pathway of Prdx6 is different from other 2-Cys family members. Similarly, another important property of Prdx6 is that it does not require thioredoxin to participate in the catalytic pathway, and glutathione (GSH) can act as a physiological reducing agent for Prdx6. The primary structure of Prdx6 consists of 224 amino acid residues, and its molecular weight ranges from $26 \mathrm{kDa}$ to $29 \mathrm{kDa}$ depending on the species (12).

Prdx6 is a bifunctional protein with both glutathione peroxidase activity (NSGPx) and phospholipase A2 activity (PLA2) (4), and exhibits 
different enzyme activities depending on the internal environment changes produced by the organisms. There are many factors affecting these two activities. It is clear from the current study that $\operatorname{Prdx} 6$ mainly exhibits NSGPx activity at $\mathrm{pH} 8(4,13)$ and PLA2 activity at $\mathrm{pH} 4$ (6). Prdx6 exhibits these activities separately mainly due to the presence of two independent active centres of Prdx6 protein, which are Ser at position 32 for PLA2 activity and Cys at position 47 for NSGPx activity (2). NSGPx activity of Prdx6 can specially be inhibited by the inhibitor mercaptosuccinate, and PLA2 activity of Prdx6 by $\operatorname{MJ} 33(5,10)$.

In our previous study, the up-regulated expression of the bifunctional Prdx6 in cells can increase the ability of Brucella to survive intracellularly (data not shown). In order to study the single function of Prdx6, open reading frames (ORFs) of Prdx6 with the single enzyme activity gene centres were designed and mutated using gene splicing by overlap extension PCR (SOE-PCR), the single active centres of Prdx6 eukaryotic expression plasmids were constructed, and the enzyme activity of single active centre Prdx6 was detected, which laid the foundation for further research on the biological function of Prdx6 with a different enzyme activity and the relationship between Prdx6 activities and Brucella infection.

\section{Material and Methods}

Biological chemicals and cells. The restriction enzymes Xba I and EcoR V, pMD18-T and Ex Taq were purchased from Takara (Kusatsu, Japan). MJ33 was purchased from Sigma Chemical Company (Sigma-Aldrich, USA). Mercaptosuccinate was sourced from Dr Ehrenstorfer GmbH (Germany). FuGENE HD Transfection Reagent was acquired from Promega (USA). Absin Bioscience (China) supplied 4-methylumbelliferyl oleate. Use was made of X5 HighFidelity DNA Polymerase PCR mix $(2 \times)$ which was a Mei5 Biotechnology-supplied product (China). The previously constructed plasmid pLenti-CMV-GFPPrdx6 with the full-length ORF of mouse Prdx6 inserted (GenBank accession number AK168223.1), the murine macrophage cell line RAW264.7, Escherichia coli DH5 $\alpha$, and pLenti-GIII CMV-GFP2A-puro plasmids (Applied Biological Materials, Canada) were available from the Institute of Zoonosis, Jilin University (China).

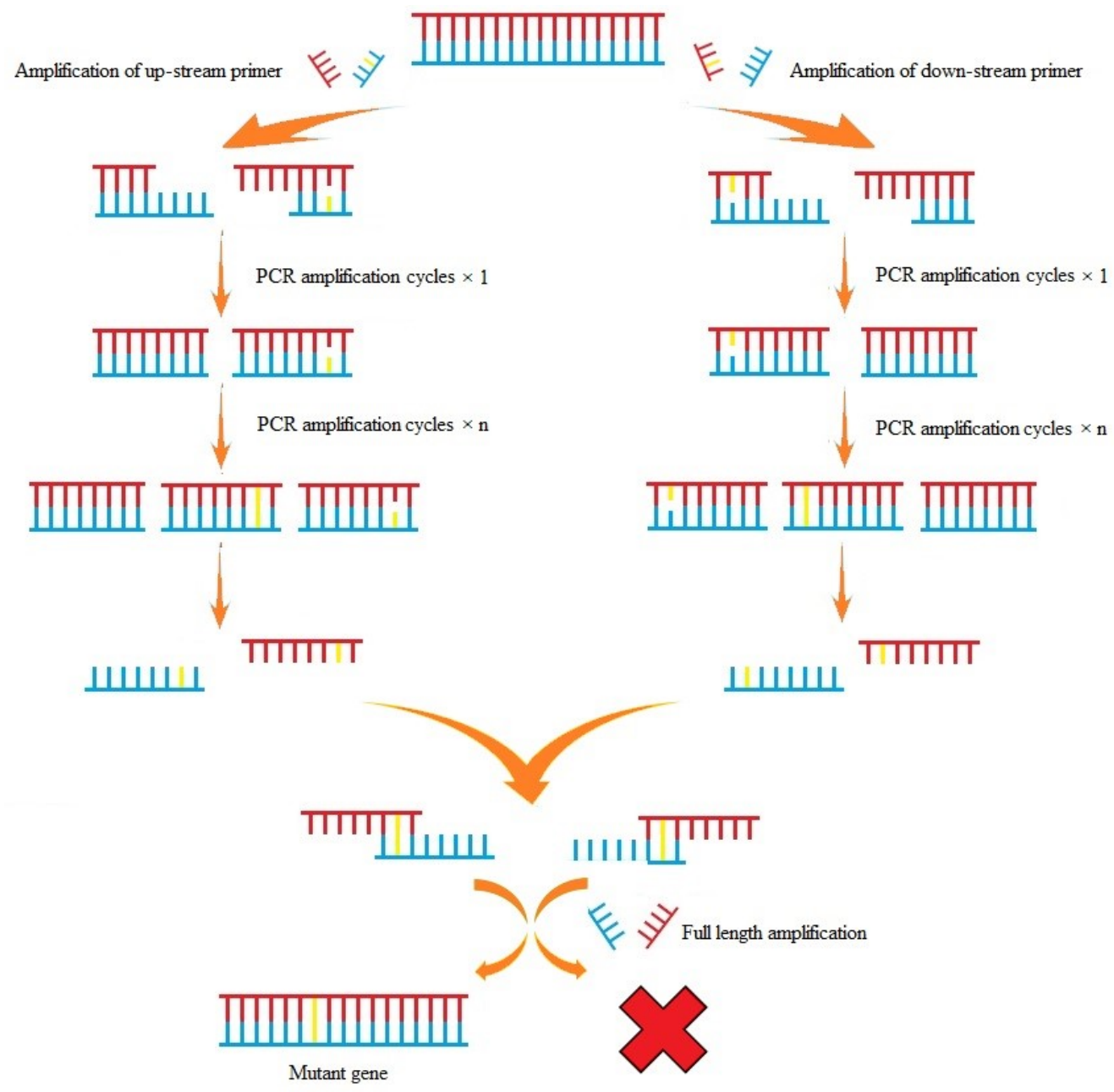

Fig. 1. The principle of SOE-PCR 
Table 1. Primers used for PCR amplification

\begin{tabular}{lll}
\hline Primer & Nucleotide sequence (5' ${ }^{\prime}$ '3') & Object \\
\hline PLA2UP-1 & ATGCCCGGAGGGTTGCTTCTC & Upstream region of Prdx6-PLA2 \\
PLA2UP-2 & CTTTACCCCAGTGTCCACCACAGAACTT & \\
PLA2LOW-1 & AAGTTCTGTGGTGGACACTGGGGTAAAG & Downstream region of Prdx6-PLA2 \\
PLA2LOW-2 & TTAAGGCTGGGGTGTATAACGGAGG & \\
NSGPxUP-1 & ATGCCCGGAGGGTTGCTTCTCG & Upstream region of Prdx6-NSGPx \\
NSGPxUP-2 & ATGCCCCATGCATCTCCCAGGAAAT & \\
NSGPxLOW-1 & ATTTCCTGGGAGATGCATGGGGCAT & Downstream region of Prdx6-NSGPx \\
NSGPxLOW-2 & TTAAGGCTGGGGTGTATAACGGAGGTATTTT & \\
Prdx6-1 & CCGGATATCATGCCCGGAGGGTTGCTT & Full length amplification \\
Prdx6-2 & TGCTCTAGATTAAGGCTGGGGTGTATAACGGAGG & \\
\hline
\end{tabular}

Primer design. Primers were designed by Primer Premier 5, following the principle of SOE-PCR (Fig. 1). Four pairs of primers (Table 1) were designed to amplify the target mutant DNA fragments upstream and downstream of Prdx6, setting the PLA2 and NSGPx active centres as the demarcation points, respectively. A pair of primers (Prdx61/Prdx62) for amplifying the full length of Prdx6 were designed, including protective bases and the restriction sites $E c o R \mathrm{~V}$ and $\mathrm{Xba}$ I for inserting the eukaryotic expression vector $\mathrm{pLenti-GIII-CMV-GFP-2A-Puro.}$

Amplification of single activity Prdx6 ORF and construction of expression vector. The eukaryotic expression pLenti-CMV-GFP-Prdx6 plasmid constructed previously in our laboratory was used as the amplification template for SOE-PCR (15). Recombinant plasmid was extracted using a TIAN prep Mini Plasmid Kit (Tiangen Biotech, China) according to the manufacturer's instructions.

Amplification was undertaken of the Prdx6 regions upstream and downstream with the centres of PLA2 and NSGPX activity as the respective demarcation points. The reaction used the following protocol. Briefly, $20 \mu \mathrm{L}$ reaction volumes containing pLenti-CMV-GFP-Prdx6 as the template (100 ng), $0.5 \mu \mathrm{L}$ of $10 \mu \mathrm{M}$ primers (Primer-up/Primer-low), $10 \mu \mathrm{L}$ of X5 High-Fidelity DNA Polymerase PCR mix, and $8 \mu \mathrm{L}$ of water were mixed. The reaction conditions were $94^{\circ} \mathrm{C}$ for $25 \mathrm{~s} ; 35$ cycles of $25 \mathrm{~s}$ at $94^{\circ} \mathrm{C}, 25 \mathrm{~s}$ at $64^{\circ} \mathrm{C}$, and $15 \mathrm{~s}$ at $68^{\circ} \mathrm{C}$; then a final extension at $68^{\circ} \mathrm{C}$ for $5 \mathrm{~min}$. The annealing temperature was the same for the four segments at $64^{\circ} \mathrm{C}$. The amplified upper and lower half PCR products were mixed directly, and the mixture diluted 100 times with sterile Milli-Q water was used as a template for the next step which was fulllength amplification of Prdx6 by means of the primer pairs Prdx61 and Prdx62. The amplification was also carried out using X5 High-Fidelity DNA Polymerase PCR mix $(2 \times)$, and the system conditions were the same as before, save for a change of annealing temperature to $62^{\circ} \mathrm{C}$. The PCR amplification products of the full-length Prdx6 ORF with the mutant nucleotide were purified by $1.0 \%$ agarose gel electrophoresis and a QIA quick DNA gel extraction kit (Qiagen, Germany), and ligated into the pMD18-T vector (Takara, China). Then, the recombinant pMD18-T with target DNA products inserted was transformed into $E$. coli $\mathrm{DH} 5 \alpha$ competent cells and identified by bacterial PCR with the primer pairs Prdx61 and Prdx62. The positive colonies were sequenced by Comate Biosciences (China). The right recombinant plasmids were subjected to double digestion of $X b a$ I and EcoR $\mathrm{V}$ restriction enzymes and subcloned into the pLenti-GIII-CMV-GFP-2A-Puro by standard molecular biology techniques. The positive colonies were sequenced.

Cell groups for activity analyses. Five groups of murine Raw264.7 macrophage cells were divided. A control group was left untransfected, and the other four groups were respectively transfected with the plasmids of pLenti-CMV-GFP-Prdx6, pLenti-CMVGFP-Prdx6-NSGPx, pLenti-CMV-GFP-Prdx6-PLA2, and pLenti-CMV-GFP-2A-Puro (empty eukaryotic expression vectors). In all groups, cells were treated with MJ33, mercaptosuccinate, or not treated with any inhibitor. Additionally, some cells in the untransfected group were treated with both MJ33 and mercaptosuccinate. FuGENE HD Transfection Reagent was used to transfect Raw264.7 cells at the ratio of 2.5:1 following the manufacturer's instructions. MJ33 in a final concentration of $20 \mu \mathrm{M}$ and mercaptosuccinate in a final concentration of $50 \mu \mathrm{M}$ were added to the cell culture plate and kept for $12 \mathrm{~h}$. Then, all the cells in the six-well plates were collected for PLA2 and NSGPx activity analysis.

Activity analysis of PLA2. The collected cells were added to $3 \mathrm{~mL}$ of $50 \mathrm{mM}$ phosphate buffer $(\mathrm{pH} 7$, $5 \mathrm{mM}$ of EDTA), agitated for $30 \mathrm{~min}$, centrifuged at $12,000 \mathrm{~g}$ for $20 \mathrm{~min}$, and the supernatant was used as an enzyme extract for viability determination. The reaction mixture consisted of $0.1 \mathrm{~mL}$ of enzyme extract, $2.8 \mathrm{~mL}$ of reaction matrix $(0.1 \mathrm{M}$ citric acid, $0.2 \mathrm{M}$ disodium phosphate, $\mathrm{NaF} 150 \mathrm{mM}, 0.8 \mathrm{mg} / \mathrm{mL}$ of bovine serum albumin, and $0.5 \mathrm{mg} / \mathrm{mL}$ of Triton X-100 at $\mathrm{pH} 5.0$ ), and $0.1 \mathrm{~mL}$ of $1 \mathrm{mM}$ 4-methylumbelliferyl oleate (fluorimetric substrate). Reaction mixtures with fluorimetric substrates were incubated at $37^{\circ} \mathrm{C}$ for $45 \mathrm{~min}$. The reaction was stopped by adding $0.5 \mathrm{~mL}$ of $1 \mathrm{M} \mathrm{HCl}$. The fluorescence value was measured at an excitation wavelength of $355 \mathrm{~nm}$ 
and an emission wavelength of $460 \mathrm{~nm}$, using an Infinite F200 Pro microplate analyser (TECAN, Switzerland) (9). This assay was repeated three times.

Activity analysis of NSGPx. NSGPx activity was measured using a Total Glutathione Peroxidase Assay Kit (Beyotime Biotechnology, China) according to manufacturer's instructions. Briefly, the collected cells in $150 \mu \mathrm{L}$ of the homogenate were homogenised in a glass homogeniser in an ice bath. The homogenate was then centrifuged at $12,000 \mathrm{~g}$ for $10 \mathrm{~min}$ at $4^{\circ} \mathrm{C}$, and the supernatant was taken for assay of enzyme activity. GPx test solution in a volume of $580 \mu \mathrm{L}(250 \mu \mathrm{L}$ of $30 \mathrm{mM}$ nicotinamide adenine dinucleotide phosphate (NADPH), $250 \mu \mathrm{L}$ of $84 \mathrm{mM} \mathrm{GSH}$, and $80 \mu \mathrm{L}$ of glutathione reductase) and $1 \mathrm{~mL}$ of $15 \mathrm{mM}$ peroxide reagent solution $(2.15 \mu \mathrm{L}$ of $\mathrm{Cum}-\mathrm{OOH}$ in $1 \mathrm{~mL}$ of Milli-Q water) were prepared in advance. The blank group prepared as $200 \mu \mathrm{L}$ reaction mixture included $185 \mu \mathrm{L}$ of glutathione peroxidase assay buffer, $11 \mu \mathrm{L}$ of GPx test solution, and $4 \mu \mathrm{L}$ of $15 \mathrm{mM}$ peroxide reagent solution. The sample group prepared as $200 \mu \mathrm{L}$ reaction mixture included $175 \mu \mathrm{L}$ of glutathione peroxidase assay buffer, $10 \mu \mathrm{L}$ of supernatant, $11 \mu \mathrm{L}$ of GPx test solution, and $4 \mu \mathrm{L}$ of $15 \mathrm{mM}$ peroxide reagent solution. OD at A340 was measured every $4 \mathrm{~min}$ and recorded continuously for $20 \mathrm{~min}$ using a microplate analyser (Biotek, USA). The unit of glutathione peroxidase activity is defined as 1 enzyme activity unit (1 unit) at $25^{\circ} \mathrm{C}$ and $\mathrm{pH} 8.0$ in $\mathrm{GSH}$, glutathione reductase and $\mathrm{Cum-OOH}$. Under the conditions present, 1 micromolar NADPH can be converted to
$\mathrm{NADP}^{+}$in $1 \mathrm{~min}$. The calculation formula for vitality is as follows :

$$
A S=\frac{A 340(S)-A 340(B)}{0.00622} \text { and } A A=\frac{A S \times \mathrm{DF}}{\mathrm{SC}}
$$

where $A S$ is glutathione peroxidase activity in the system, $A 340(S)$ is $\mathrm{A} 340 / \mathrm{min}$ (sample), $A 340(B)$ is A340/min (blank); $A A$ is glutathione peroxidase activity in the sample, DF is dilution factor, and SC is protein concentration in the sample.

This assay was repeated three times.

Statistical analysis. Statistical analysis was conducted by two-way analysis of variance (ANOVA) using SPSS 13.0 software (IBM SPSS, USA).

\section{Results}

Construction of eukaryotic expression vector. The sequencing results were compared with Prdx6 DNA (GenBank accession number AK168223.1) by DNAMAN 6.0 (Lynnon Biosoft, USA) (Fig. 2) where it was seen that the $94^{\text {th }}$ nucleotide $T$ was changed to $G$ and the $140^{\text {th }}$ nucleotide $\mathrm{G}$ was changed to $\mathrm{C}$, confirming the successful construction of a single activity centre Prdx6 expression plasmid. The amino acid mutation sequence of Prdx6 were compared (GenBank accession number BAE40177.1) by ClustalX (Conway Institute, University College Dublin, Ireland) (Fig. 3), and the Ser32 was observed to have mutated to Ala32 and the Cly47 to Ser47 by changing the corresponding basis.

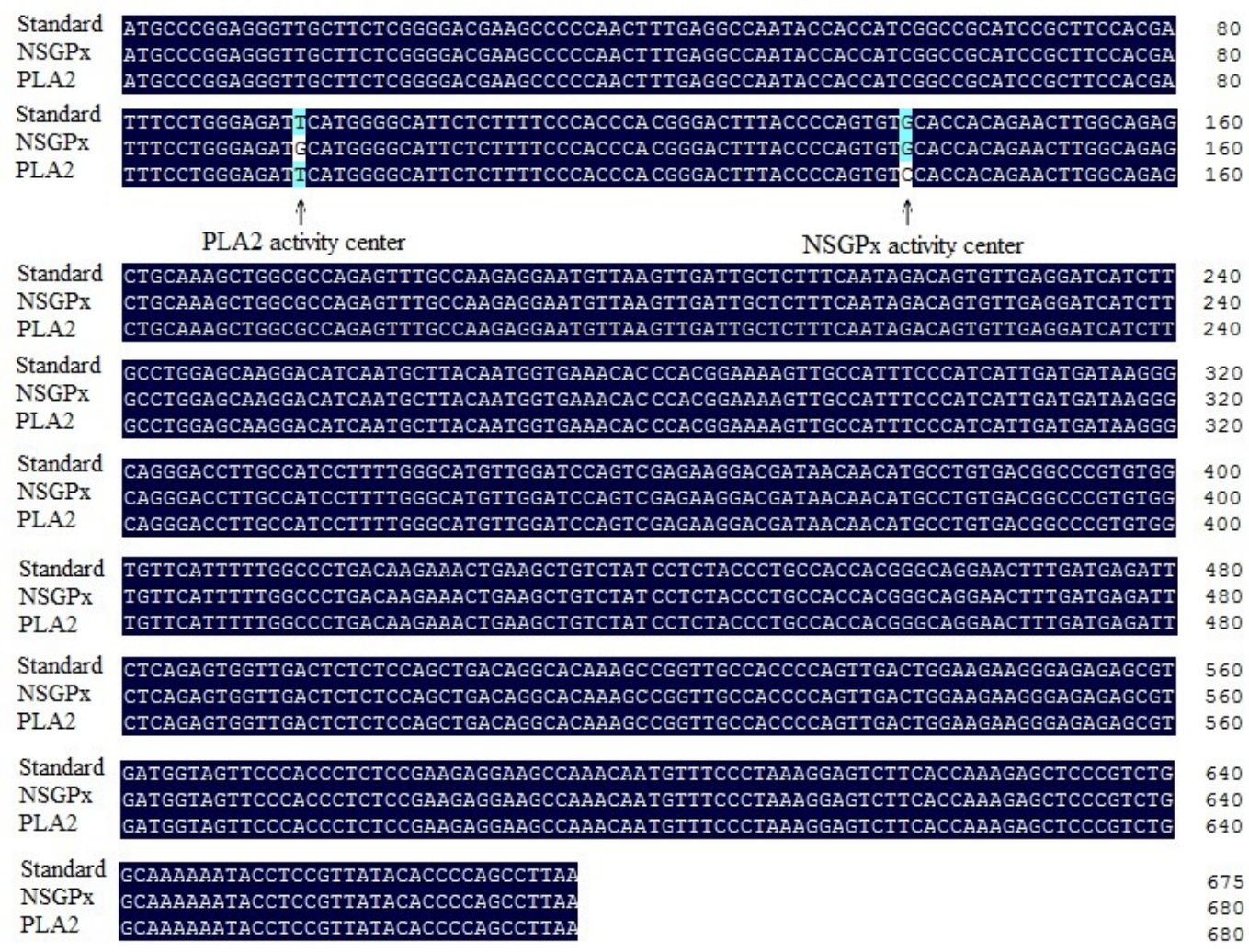

Fig. 2. Sequencing results of the single-function Prdx6 DNA. NSGPx - Prdx6 with the NSGPx single active centre. PLA2 Prdx6 with the PLA2 single active centre. Standard - original Prdx6 ORF sequence (GenBank accession number AK168223.1) 

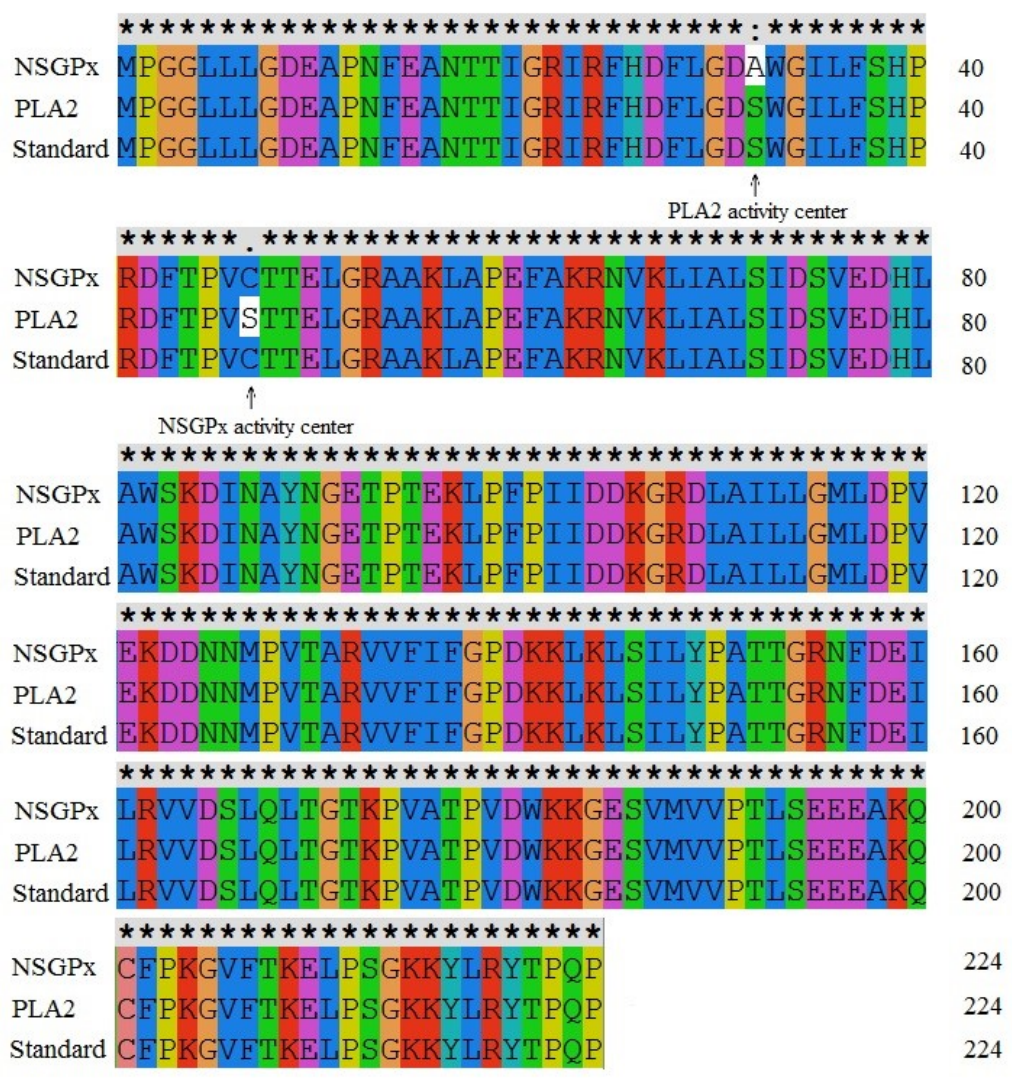

Fig. 3. Amino acid mutation of Prdx6 protein. Denotations as in Fig. 2. Standard the original Prdx6 amino acid sequence (GenBank accession number BAE40177.1)

PLA2 activity analysis. 4-Methylumbelliferyl oleate as a substrate was decomposed into 4-methylumbelliferone by PLA2 to produce a fluorescence signal. It follows that the stronger the measured fluorescence value is, the stronger the activity of PLA2 is in the sample. Fig. 4 presents PLA2 activity graphically. The pink and blue columns in the PLA2-transfected group are higher than the untransfected, the empty vector-transfected, and NSGPx-transfected groups. This indicates that the NSGPx active centre mutated in the pLenti-CMV-GFPPRDX6-PLA2 plasmid did not affect the PLA2 activity. Fig. 5 presents NSGPx activity graphically. The pink and yellow columns in the untransfected, empty vector-transfected, and PLA2-transfected groups are significantly lower than those in the Prdx6transfected and the NSGPx-transfected groups. This indicates that the NSGPx active centre in the pLentiCMV-GFP-PRDX6-PLA2 plasmid was mutated successfully. In the graphic for PLA2 activity (Fig. 4), the yellow column is generally lower than the pink and blue ones in each group, and in overexpressed groups (Prdx6-transfected and PLA2-transfected groups), this indicates that the PLA2 activity is well suppressed by MJ33. The height of the green column is similar to that of the yellow column, indicating that mercaptosuccinate has no effect on MJ33, and MJ33 still inhibits PLA2 activity.

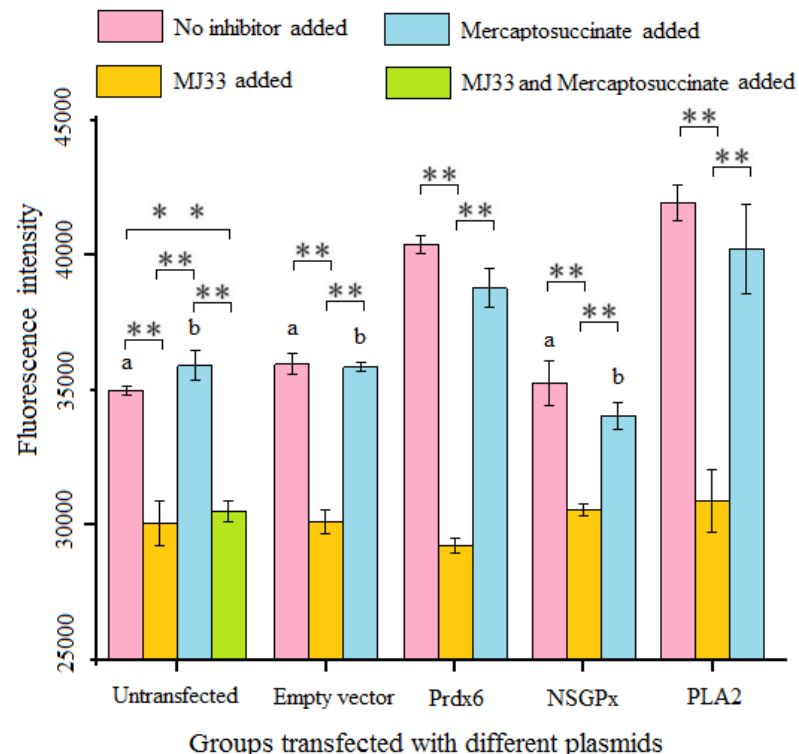

Fig. 4. PLA2 activity analysis. Untransfected - Raw 264.7 cells without any constructed plasmids. Empty vector - Raw264.7 cells transfected with pLenti-CMV-GFP-2A-Puro plasmids. Prdx6 Raw264.7 cells transfected with pLenti-CMV-GFP-Prdx6 plasmid. NSGPx - Raw264.7 cells transfected with pLenti-CMV-GFP-Prdx6NSGPx plasmid. PLA2 - Raw264.7 cells transfected with pLentiCMV-GFP-Prdx6-PLA2

Statistical analysis was conducted by two-way analysis of variance using SPSS 13.0 software. * $\mathrm{P}<0.05, * * \mathrm{P}<0.01 .{ }^{\mathrm{a}} \mathrm{P}<0.05$ vs. the pink column in PLA2 group, ${ }^{\mathrm{b}} \mathrm{P}<0.05$ vs. blue column in PLA2 group 
NSGPx activity analysis. The pink and yellow columns in NSGPx are higher than untransfected empty vector and PLA2. This indicates that the PLA2 active centre mutated in the pLenti-CMV-GFP-PRDX6NSGPx plasmid did not affect the NSGPx activity (Fig. 5). Whereas, as Fig. 4 shows, the pink and blue columns in groups of the untransfected, empty vector and NSGPx are significantly lower than those in the groups of the Prdx6 and PLA2. It indicates that the PLA2 active centre in the pLenti-CMV-GFP-PRDX6NSGPx plasmid was mutated successfully. The blue columns are generally lower than the pink and yellow columns in each group and in overexpressed groups (Prdx6-transfected and NSGPx-transfected groups). This indicates that the NSGPx activity was well suppressed by mercaptosuccinate (Fig. 5). The height of the green column is similar to that of the blue column, indicating that MJ33 has no effect on mercaptosuccinate, and mercaptosuccinate still inhibits NSGPx activity (Fig. 5).

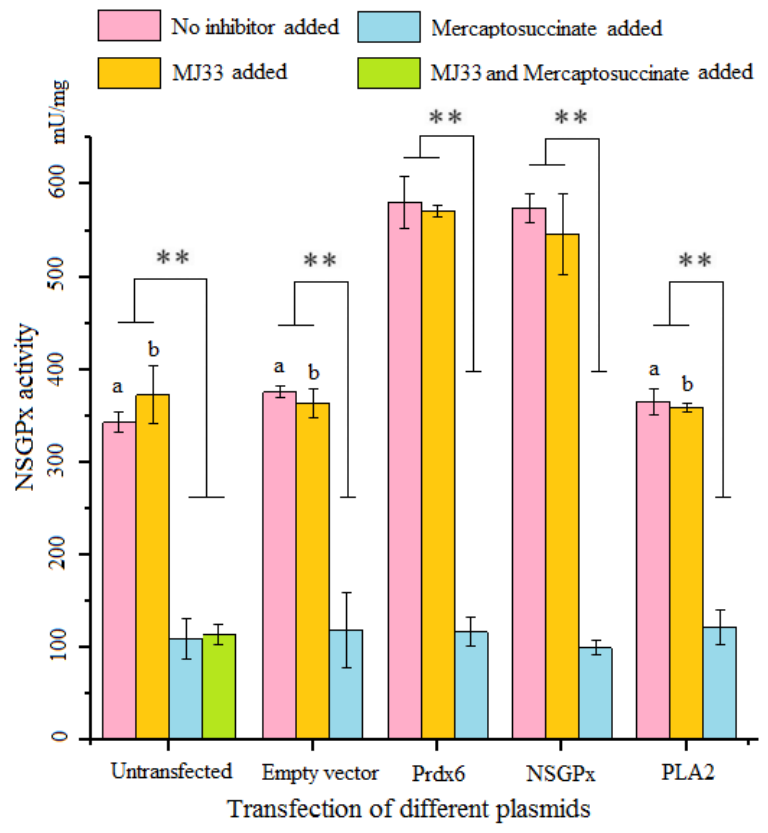

Fig. 5. NSGPx activity analysis. Untransfected - Raw 264.7 cells without any constructed plasmids. Empty vector - Raw264.7 cells transfected with pLenti-CMV-GFP-2A-Puro plasmids. Prdx6 Raw264.7 cells transfected with pLenti-CMV-GFP-Prdx6 plasmid. NSGPx - Raw264.7 cells transfected with pLenti-CMV-GFP-Prdx6NSGPx plasmid. PLA2 - Raw264.7 cells transfected with pLentiCMV-GFP-Prdx6-PLA2

Statistical analysis was conducted by two-way analysis of variance using SPSS 13.0 software. $* \mathrm{P}<0.05, \quad * * \mathrm{P}<0.01 .{ }^{\mathrm{a}} \mathrm{P}<0.05$ vs. the pink column in the NSGPx group; ${ }^{\mathrm{b}} \mathrm{P}<0.05 v s$. the yellow column in the NSGPx group

\section{Discussion}

Previous studies have found that when Brucella of different virulence infect mouse Raw264.7 macrophage, no different expression of Prdx6 is caused (13). They also demonstrated that the presence of
Prdx6 has a positive effect on the intracellular survival of Brucella. At present, whether the single PLA2 activity, the single NSGPx activity or both PLA2 and NSGPx activities of Prdx6 play a major role in the intracellular survival process of Brucella is not clear. Gaining clarity on these issues will greatly assist our in-depth study of Brucella infection in the future.

Prdx6 is an important anti-damage protein in the host. Because of its two different biological activities, Prdx6 exerts different effects under different conditions (7). When foreign substances invade the body, the host will produce reactive oxygen species (ROS) to nonspecifically remove that invasion in order to resist the damage wrought by the foreign substances. However, the killing effect of ROS is non-specific, it will also cause damage to the host itself. The NSGPx activity of Prdx6 can act on ROS after ROS is elevated, reducing the damage caused by ROS to the body (14). NSGPx showed the greatest activity at $\mathrm{pH} 8$. Prdx6 can also exhibit non- $\mathrm{Ca}^{2+}$-dependent PLA2 activity. Its main function is to participate in energy metabolism and maintain cell membrane balance. PLA2 exerts maximum activity at $\mathrm{pH} 4(1,2)$.

In this study, the recombinant expression plasmid pLenti-CMV-GFP-Prdx6 constructed previously was used as a template instead of cDNA from total RNA, which simplified the experiment and improved the probability of obtaining the target fragment. The target fragment for the upstream and downstream of Prdx6 with only a PLA2 active centre can be obtained using two pairs of primers (PLA2UP-1/PLA2UP-2 and PLA2DOWN-1/PLA2DOWN-2) (Table 1) for mutating the $\mathrm{Ser}^{32}$ to $\mathrm{Ala}^{32}$ by changing the $94^{\text {th }}$ nucleotide from T to G. So that, Prdx6 loses the PLA2 active centre, but retains its NSGPx active centre. Similarly, Prdx6 with only an NSGPx active centre was constructed using two pairs of primers (NSGPxUP1/NSGPxUP-2 and NSGPxLOW-1/NSGPxLOW-2) (Table 1) to mutate the $\mathrm{Cly}^{47}$ to $\mathrm{Ser}^{47}$ by changing the $140^{\text {th }}$ nucleotide from $\mathrm{G}$ to $\mathrm{C}$, leading to Prdx6 losing the NSGPx active centre and retaining its PLA2 active centre. Since the different $\operatorname{Prdx} 6$ proteins were expressed in the cells, the restriction enzyme sites $X b a \mathrm{I}$ and $E c o R \mathrm{~V}$ were selected to construct the recombinant eukaryotic expression plasmids, ensuring that the size of the recombinant protein expressed in the eukaryotic is closer to the size of the target protein with fewer fusion proteins from the carrier, so that the conformation of the obtained protein is closer to the native protein.

Several studies in which the inhibitor mercaptosuccinate of NSGPX and inhibitor MJ33 of PLA2 were used, have confirmed that Prdx6 possesses both PLA2 and NSGPx activities with separate activity centres (3). However, the analysis based on the biofunction of Prdx6 cannot rule out the incompleteness of the inhibitor. In this experiment, respectively through mutating the NSGPx active centre and PLA2 active centre of Prdx6 to construct the single 
enzyme activity of Prdx6, the two biological activities of Prdx6 were fully demonstrated by their verification with a single active centre at the levels of the inhibition and overexpression. This further confirmed the reliability of PLA2 and NSGPx activity of Prdx6 and has laid a solid foundation for the next study of the relationship between the single-function enzyme activity of Prdx6 and Brucella infection.

Conflict of Interest Statement: The authors declare that there is no conflict of interests regarding the publication of this article.

Financial Disclosure Statement: This work was supported by the grants from the National Key R\&D Program of China (Nos. 2018YFD0500900 and 2018YFD0500903), the Open Funds of the State Key Laboratory of Veterinary Etiological Biology, Lanzhou Veterinary Research Institute, Chinese Academy of Agricultural Sciences (No. SKLVEB2017KFKT006), and the Science and Technology Development Project of Jilin Province, China (No. 20170101149JC).

Animal Rights Statement: None required.

Acknowledgements: Wang L.L. (1lw1215@foxmail. com) and Lu S.Y. (lushiying1129@163.com) made equal contributions to this study. Ren H.L. (renhl@jlu.edu.cn) is the corresponding author.

\section{References}

1. Benipal B., Feinstein S.I., Chatterjee S., Dodia C., Fisher A.B.: Inhibition of the phospholipase A2 activity of peroxiredoxin 6 prevents lung damage with exposure to hyperoxia. Redox Biol 2015, 4, 321-327.

2. Chatterjee S., Feinstein S.I., Dodia C., Sorokina E., Lien Y.C., Nguyen S., Debolt K., Speicher D., Fisher A.B.: Peroxiredoxin 6 , phosphorylation, and subsequent phospholipase $\mathrm{A}_{2}$ activity are required for agonist-mediated activation of NADPH oxidase in mouse pulmonary microvascular endothelium and alveolar macrophages. J Biol Chem 2011, 286, 11696-11706.
3. Chhunchha B., Kubo E., Fatma N., Singh D.P.: Sumoylationdeficient Prdx6 gains protective function by amplifying enzymatic activity and stability and escapes oxidative stressinduced aberrant Sumoylation. Cell Death Dis 2017, 8, $2525-2525$.

4. Fisher A.B.: Peroxiredoxin 6: a bifunctional enzyme with glutathione peroxidase and phospholipase $\mathrm{A}_{2}$ activities. Antioxid Redox Signal 2011, 15, 831-844

5. Fisher A.B., Dodia C.: Role of acidic $\mathrm{Ca} 2+$-independent phospholipase A2 in synthesis of lung dipalmitoyl phosphatidylcholine. Am J Physiol-Lung Cell Molec Physiol 1997, 272, 238-243.

6. Fisher A.B., Dodia C., Feinstein S.I., Ho Y.S.: Altered lung phospholipid metabolism in mice with targeted deletion of lysosomal-type phospholipase $\mathrm{A}_{2}$. J Lipid Res 2005, 46, 1248-1256.

7. Fisher A.B., Dodia C., Manevich Y., Chen J.W., Feinstein S.I.: Phospholipid hydroperoxides are substrates for non-selenium glutathione peroxidase. J Biol Chem 1999, 274, 21326-21334.

8. Fisher A.B., Dodia C.: Role of phospholipase A2 enzymes in degradation of dipalmitoylphosphatidylcholine by granular pneumocytes. J Lipid Res 1996, 37, 1057-1064.

9. Hernández P., Zomeño L., Ariño B., Blasco A.: Antioxidant, lipolytic and proteolytic enzyme activities in pork meat from different genotypes. Meat Sci 2004, 66, 525-529.

10. Jo M., Yun H.M., Park K.R., Hee P.M., Myoung K.T., Ho P.J., Jae L.S., Moon D.C., Park C.W., Song S., Lee C.K., Bae H.S., Tae H.J.: Lung tumor growth-promoting function of peroxiredoxin 6. Free Radical Biol Med 2013, 61, 453-463.

11. Liu N.N., Liu Z.S., Lu S.Y., Hu P., Li Y.S., Feng X.L., Zhang S.Y., Wang N., Meng Q.F., Yang Y.J., Tang F., Xu Y.M., Zhang W.H., Guo X., Chen X.F., Zhou Y., Ren H.L.: Full-length cDNA cloning, molecular characterization and differential expression analysis of peroxiredoxin 6 from Ovis aries. Vet Immunol Immunopathol 2015, 164, 208-219.

12. Liu N.N., Liu Z.S., Lu S.Y., Hu P., Zhang Ying, Fu B.Q., Li Y.S., Zhou Y., Zhang Yu, Ren H.L.: Isolation and characterisation of peroxiredoxin 6 promoter from sheep (Ovis aries). J Vet Res 2016, 60, 315-321.

13. Manevich Y., Fisher A.B.: Peroxiredoxin 6, a 1-Cys peroxiredoxin, functions in antioxidant defense and lung phospholipid metabolism. Free Radical Biol Med 2005, 38, 1422-1432.

14. Wu Y.Z., Manevich Y., Baldwin J.L., Dodia C., Yu K., Feinstein S.I., Fisher A.B.: Interaction of surfactant protein A with peroxiredoxin 6 regulates phospholipase $A_{2}$ activity. J Biol Chem 2006, 281, 7515-7525.

15. Zhao C., Zhao J., Wang W., Fan Y., Ma C., Zhang D., Lv Y.: Expression of MLAA34-HSP70 fusion gene constructed by SOE-PCR. Pakistan J Sci 2017, 30, 1125. 